

\title{
ANALOGÍAS COMO ESTRATEGIA PARA LOGRAR LA COMPRENSIÓN DE LA SELECCIÓN NATURAL*
}

\section{Analogies as a Strategy to Achieve the Understanding of Natural Selection}

\section{Analogias como Estratégia para Alcançar a Compreensão da Seleção Natural}

\author{
Juan Sebastián Palacios-Rodríguez **

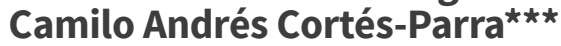

Fecha de recepción: 7 de septiembre de 2019

Fecha de aceptación: 29 de mayo de 2020

\section{Cómo citar este artículo:}

Palacios-Rodríguez, J. S. y Cortés-Parra, C. A. (2020). Analogías como estrategia para lograr la comprensión de la selección natural. Bio-grafía, 13(25). 143-151. https://doi.org/10.17227/bio-grafia.vol.13.num25-12413

\section{Resumen}

Las analogías han sido descritas como herramientas de gran utilidad en el desarrollo de conceptos explicativos, ya que permiten comprender determinados fenómenos a través de su relación con un sistema análogo. En este artículo de investigación se desarrolla una analogía como estrategia de aprendizaje para facilitar la comprensión de la selección natural mediante una actividad en la cual se integran diferentes elementos conceptuales de la temática general, en el marco de la aplicación de una unidad didáctica enfocada en la enseñanza de y desde la teoría evolutiva para estudiantes de séptimo grado del Colegio Panamericano (IED), Bogotá, Colombia. Los participantes respondieron positivamente ante la analogía, ya que comprendieron los elementos teóricos involucrados y fueron capaces de asociarlos con los conceptos vistos en clases teóricas previas. Por último, los conectaron con la explicación del mecanismo de selección natural que fue integrado correctamente en sus argumentaciones posteriores. La analogía se implementó de forma eficaz como herramienta complementaria y de transversalización en la enseñanza de la evolución por selección natural; se obtuvieron resultados satisfactorios en la comprensión de conceptos que resultan particularmente difíciles de entender para los estudiantes.

Palabras clave: conceptos; didáctica; enseñanza; evolución; transversalización

* Queremos agradecer a los estudiantes de séptimo grado y directivos del Colegio Panamericano IED por su disposición, consentimiento y participación en el desarrollo de este trabajo. Asimismo, agradecemos al doctor Guillermo Fonseca por sus asesorías, correcciones y sugerencias durante la elaboración y planificación de las clases, también a Juan Camilo Ríos por sus comentarios y aportes a la versión final del presente artículo.

** Estudiante de Licenciatura en Biología de la Universidad Distrital Francisco José de Caldas. Correo electrónico: juspalaciosr@udistrital.edu.co. Orcid: https://orcid.org/0000-0001-5109-9520

*** Estudiante de Licenciatura en Biología de la Universidad Distrital Francisco José de Caldas. Correo electrónico: cacortesp@udistrital.edu.co. Orcid: https://orcid.org/0000-0002-1229-8233 


\section{Abstract}

Analogies have been described as very useful tools in the development of explanative concepts, because they enable understanding certain phenomena through their relationship with an analogous system. In this paper, the authors developed an analogy as a strategy to facilitate the comprehension of natural selection. They carried out an activity that integrates different conceptual elements of the topic, within the framework of the application of a didactic unit for seventh grade students of the Colegio Panamericano (IED), Bogotá, Colombia, focused on the teaching of/from the theory of evolution. The students responded positively to the analogy. They understood the theoretical elements involved in it and were able to associate them with the concepts discussed in previous lessons. Finally, they associated those concepts to the explanation of the mechanism of natural selection, which correctly integrated into their subsequent arguments. The analogy was effectively implemented as a complementary and cross-curricular tool in the teaching of evolution. The results obtained in the understanding of concepts that are particularly difficult for students were satisfying.

Keywords: concepts; didactics; teaching; evolution; mainstreaming

\section{Resumo}

As analogias têm sido descritas como ferramentas muito úteis no desenvolvimento de conceitos explicativos, pois permitem compreender determinados fenômenos por meio de sua relação com um sistema análogo. A seguir, desenvolve-se uma analogia como estratégia que permite facilitar a compreensão da seleção natural através de uma atividade realizada com aviões de papel, no âmbito da aplicação de uma unidade didática na teoria da evolução e seus elementos conceituais para alunos do sétimo ano em Colegio Panamericano (IED), Bogotá, Colombia. Os alunos responderam positivamente à analogia, compreendendo os elementos teóricos envolvidos e podendo associá-los aos conceitos apresentados nas aulas teóricas anteriores, para finalmente ligá-los à explicação do mecanismo de seleção natural que foi corretamente integrado nos seus argumentos subsequentes. A analogia foi efetivamente implementada como uma ferramenta complementar e de integração no ensino da evolução por seleção natural, obtendo resultados satisfatórios na compreensão de conceitos que são particularmente difíceis para os alunos entenderem.

Palavras-chave: conceitos; didática; ensino; evolução; transversalidade 
La teoría evolutiva es central, estructural y fundamental en los procesos de enseñanza y aprendizaje de la biología por cuanto logra fortalecer la formación de ciudadanos científicamente alfabetizados y promover una adecuada comprensión de los fenómenos biológicos (Chaves Mejía, 2016; Gagliardi, 1986). En este sentido, se considera que la evolución es un concepto estructurante, ya que se integra con otros para producir una unidad más compleja que organiza y da sentido a los conocimientos que va adquiriendo el estudiante, actuando en todos los campos de la biología como una teoría unificadora (Castro y Valbuena, 2007; Gagliardi, 1986; Chaves Mejía, 2016).

Dada su complejidad, la enseñanza de esta teoría requiere que los conocimientos previos sean articulados por el estudiante para lograr una comprensión adecuada. El docente debe tener especial cuidado con los diferentes conceptos que integran y dan sentido a la teoría evolutiva, para así afrontar las tres formas de pensar que se consideran obstáculos en la enseñanza de la evolución: la teleología, el razonamiento centrado en el individuo y el razonamiento causal lineal (González Galli y Meinardi, 2015). Sin embargo, actualmente la evolución se enseña a través de un conjunto de conceptos aislados e inconexos sobre herencia, variación, diversidad y selección (Marcos Merino et al., 2017). Tratar aisladamente los conceptos involucrados limita el entendimiento de la evolución y su articulación con los demás saberes biológicos. La especiación y la selección natural se destacan como los pilares principales que deben ser obligatorios en la enseñanza de la evolución (González Galli, 2015; Van Dijk y Reydon, 2010), pero resultan ser particularmente complejos para los estudiantes.

Son múltiples las formas de enseñar esta teoría científica en el aula, y no es difícil encontrar actividades, estrategias y contenidos dirigidos a la apropiación del concepto evolutivo (Hernández et al., 2009). Las narraciones históricas, la observación, el trabajo de campo y la modelización han sido de gran importancia en la enseñanza y la comprensión de la biología y la evolución, teniendo en cuenta marcos teóricos experimentales y referenciales (Castro y Valbuena, 2007). Entre las estrategias para contrarrestar los obstáculos que surgen a la hora de enseñar evolución se destaca el uso de las ideas previas, la implementación de modelos conceptuales y el uso de las analogías (González Galli, 2015; Jiménez, 1991; Martínez Arango y Sanabria Totaitive, 2018; Oliva et al., 2001).

Las analogías, particularmente, han sido descritas por muchos autores como estrategias poderosas que pueden contribuir al desarrollo de conceptos explicativos (Cf. Glynn y Takahashi, 1998; Oliva et al., 2001; Oliva et al., 2003; Oliva y Aragón, 2009), ya que permiten comprender un fenómeno determinado que ha sido denominado como blanco, objeto o problema a través de las relaciones que se establecen con un sistema análogo (Oliva et al., 2001). Las analogías podrían ser incluso representaciones de modelos, siempre que permitan, a quien las usa, describir, explicar, predecir e intervenir, mientras no se reduzcan a aproximaciones superficiales del objeto en cuestión (Adúriz Bravo, 2010).

Los mecanismos del aprendizaje por analogía parten de una noción previa del alumno sobre el blanco, una noción sobre el análogo y un conjunto de recursos didácticos implementados por el docente a lo largo de sus explicaciones (Oliva, 2007). Estos recursos se implementan para desencadenar el pensamiento analógico en el estudiante, de tal manera que permita construir vínculos entre su conocimiento, experiencias pasadas y los nuevos contextos, contenidos o problemas planteados (Oliva et al., 2001). Si bien las analogías permiten explicar un concepto particular, deben relacionarse con información previa, que debe ser uno de los elementos que la conforman.

Cabe resaltar que las analogías ya han sido utilizadas como estrategia en la enseñanza de conceptos evolutivos con resultados satisfactorios. Marcos Merino et al. (2017) proponen explicar los relojes moleculares a partir de las diferentes variaciones que ha tenido el Quijote a lo largo de sus diferentes ediciones; Martínez Arango y Sanabria Totaitive (2018) relacionan la evolución biológica con un tema bastante familiar en el que están inmersos los estudiantes en la actualidad: los videojuegos; Naranjo Cardona (2013) usa las analogías con el fin de facilitar la comprensión de los procesos de estratificación y formación de fósiles, de manera que son los estudiantes quienes establecen las relaciones existentes entre los elementos de la analogía propuesta y los elementos de la temática blanco. Así, las analogías representan una estrategia potencial para explicar los fenómenos evolutivos y los conceptos base que forman parte de su explicación, como la selección natural. El uso de las analogías se hace tan necesario que incluso el mismo Darwin utilizó los principios de la selección artificial como analogía para explicar los fundamentos de su propuesta sobre la selección natural (Chaves Mejía, 2012). Es necesario que los docentes compartan y divulguen las herramientas, experiencias y estrategias inéditas utilizadas en el aula, de manera que conceptos como la evolución posean una amplia gama de material bibliográfico disponible y que otros docentes puedan usar para desenvolverse en diferentes contextos, problemáticas y necesidades.

En este trabajo se propone la realización de una analogía diseñada por los autores, como estrategia complementaria con el fin de facilitar la comprensión de la selección natural en el marco de la implementación de una unidad 
didáctica titulada "Adaptemos nuestra visión de la ciencia. Comprensión de la diversidad a la luz de la evolución", la cual fue aplicada a 20 estudiantes de séptimo grado de un colegio de Bogotá. Se describe la analogía y su fundamento, para que pueda ser replicada, transformada o usada como guía por otros docentes en el marco de la enseñanza de la teoría evolutiva y conceptos afines.

\section{Metodología}

De acuerdo con la estructura de la analogía realizada por Oliva (2008), se elaboró una analogía como estrategia complementaria que permitió a los estudiantes entender adecuadamente el proceso de selección natural. Esta fue desarrollada en el marco de una unidad didáctica cuyo enfoque era la enseñanza de la teoría evolutiva con el fin de comprender la diversidad biológica, con la selección natural como principal mecanismo de cambio, implementada en el Colegio Panamericano IED de Bogotá (Colombia) con 20 estudiantes de séptimo grado siguiendo los principios del modelo pedagógico de investigación dirigida (Pozo, 1997) durante un periodo de tres meses. En este modelo pedagógico, el estudiante es el centro del quehacer docente y este último actúa como un director de investigación (Pozo, 1997). Así, a lo largo del desarrollo de la unidad didáctica, cada concepto se complementa con una actividad, ya sea un laboratorio, una actividad descriptiva o -como en este caso- una analogía dirigida por el docente, en la que el estudiante se enfrenta a un problema desde un contexto de la actividad científica. Es decir, se aborda la resolución de un problema desde la búsqueda de respuestas a los fenómenos evolutivos, se cuestiona el sentido de la actividad mientras se desarrolla y se vincula con los conocimientos adquiridos y con los demás conceptos que se integran sucesivamente a la temática general. La analogía se establece como herramienta complementaria en la comprensión de la selección natural debido a su potencial explicativo y a la capacidad de involucrar diferentes conceptos como elementos análogos, a la vez que permite transversalizar con una actividad cotidiana y solventar la incapacidad de evidenciar experimentalmente el fenómeno real dentro del aula. Durante la implementación de la actividad se tomaron registros de audio de cada una de las sesiones, como evidencia y como material de análisis posterior, con el fin de captar todos los elementos posibles, identificar falencias de los estudiantes en la comprensión de los diferentes conceptos y posibles errores de los docentes durante el desarrollo de las clases.

\section{Desarrollo de la analogía}

Como bien se mencionó, la actividad está incluida en el desarrollo de una unidad didáctica dividida en módulos.
En el tercero se desarrollan los conceptos de mutación, variación y selección, por lo que previo a la realización de la actividad, a los estudiantes se les explicaron de manera teórica los fundamentos de la selección natural y los conceptos involucrados (adaptación, variación, población, individuo, mutación, etc.). En este módulo, además de la actividad central, se desarrollaron explicaciones centradas en ejemplos, tales como la evolución del cuello de la jirafa desde una visión no lamarckiana, los cambios en la frecuencia de fenotipos durante varias generaciones en una población de escarabajos sujeta a determinadas presiones de selección, entre otros. La analogía sintetiza y unifica dichas temáticas, por lo que el objetivo fue implementarla como complemento de las clases, fortaleciendo la explicación y poniendo en evidencia el nivel de comprensión de los estudiantes.

La actividad se tituló “¡Una carrera para sobrevivir!” y está centrada en el desarrollo de una analogía con aviones de papel dividida en tres partes:

1. Cada estudiante elaboró un avión de papel de la forma que consideró más apropiada; luego se les pidió realizar una fila hombro con hombro para arrojar sus aviones. Los estudiantes que no pudieron o no sabían elaborar el avión fueron eliminados de la carrera.

2. Se seleccionaron los cuatro aviones que mostraron mejor desempeño en el vuelo (aquellos que llegaron más lejos). Quienes elaboraron dichos aviones seleccionados enseñaron a sus compañeros cómo realizarlos (a cada uno se le asignó un número igual de estudiantes).

3. Después de que cada estudiante construyó su avión como le enseñó su compañero, se les indicó que debían agregarles algo novedoso (por ejemplo, un pliegue de más, un agujero, etc.) y se procedió a lanzarlos nuevamente, observando a continuación los cuatro aviones que lograron una distancia de vuelo mayor.

Durante la actividad, y al final de esta, se formularon preguntas a los estudiantes sobre lo que comprendían, cómo se vinculaba la carrera de aviones con los conceptos vistos en clase y su relación con los elementos blanco.

\section{Análisis de la actividad}

Previo a su implementación, se presentaron las actividades dentro de la unidad didáctica a docentes y compañeros de carrera para su validación. La unidad didáctica en general y sus actividades fueron revisadas minuciosamente por un docente de área antes, durante y después de su desarrollo. Para la analogía, se establecieron los elementos 
que constituyen el análogo con relación a los elementos que conforman el blanco (véase la tabla 1) teniendo en cuenta que la selección natural es un fenómeno de cambio evolutivo que se explica bajo tres premisas: 1) el rasgo sujeto a selección debe ser heredable; 2) debe existir variabilidad del rasgo entre los individuos de una población; 3) la variabilidad del rasgo debe dar lugar a diferencias en la supervivencia o el éxito reproductivo y provocar que algunas características de nueva aparición se extiendan en la población (Naranjo Cardona, 2013).

Como resultado de la intervención pedagógica se obtuvieron las grabaciones de audio de las clases, que se transcribieron a un documento para su análisis. Además, se tomaron apuntes en el diario docente durante y después de las clases con observaciones sobre las actividades realizadas y la participación de los estudiantes. Se identificaron las falencias de la analogía con relación al blanco, y se resaltaron los aspectos en los cuales esta no daba cuenta de la totalidad del fenómeno, lo cual ha sido descrito por Oliva et al. (2001) como un ejercicio necesario, ya que la analogía no debe ser completamente igual al blanco, pero tampoco muy diferente, de lo contrario el estudiante podría no encontrar la relación entre ambos sistemas.

El análisis de la respuesta de los estudiantes a la analogía se llevó a cabo desde una perspectiva inmersa en la investigación cualitativa, considerando las diferentes evidencias obtenidas a partir de la observación, las transcripciones escritas y las notas del diario docente; también, revisando de forma minuciosa sus respuestas en la evaluación de final de curso.

\section{Resultados y análisis}

La analogía resulta ser una herramienta con gran potencial explicativo en conceptos difíciles de comprendery de plasmar experimentalmente dentro del aula, tal como la selección natural. Además, se complementa con el enfoque pedagógico, ya que el estudiante se desenvuelve en una actividad indirectamente relacionada con la temática en búsqueda de las respuestas y explicaciones, desarrollando su capacidad de implementar y aplicar lo aprendido en diferentes contextos y no solo dentro del aula. La analogía fue considerada una estrategia complementaria debido a que se desarrolló después de explicaciones generales del tema, e integradora porque abarcaba otros aspectos vistos antes (variabilidad, adaptación y mutación). En el momento de implementarla es necesario comprender que la evolución biológica implica una variedad de procesos naturales en varios niveles organizacionales: mutación y recombinación a nivel genético; selección natural a nivel de individuo; deriva genética y adaptación a nivel poblacional; y especiación a nivel de especie (van Dijky Reydon,
2010). Por lo que, en primera medida, se asumió que todos los aviones de papel presentes en el grupo representaban una población sujeta a presiones de selección locales, y cada avión era un individuo miembro de esa población; de esta manera, la selección actúa a nivel de individuo, pero el cambio adaptativo a nivel de población (van Dijk y Reydon, 2010).

Se debe hacer la salvedad de que cuando aparece una presión de selección todos los individuos compiten entre sí para sobrevivir, pero aquellos que no presenten las adaptaciones necesarias serán eliminados por selección natural. Estas adaptaciones surgen aleatoriamente, producto de mutaciones genéticas. En esta analogía, los estudiantes elaboraron individualmente los aviones de papel, sin embargo, no se les dijo que su desempeño en el vuelo sería puesto a prueba, de esta forma lo realizaron como quisieron, con los criterios que ellos mismos consideraron necesarios (tamaño, estética, velocidad, etc.). Esta diversidad en los diseños es análoga a la variabilidad intraespecífica que encontramos en la naturaleza, ya que los individuos de una población pueden variar respecto a un carácter cualquiera, siendo prácticamente imposible encontrar dos individuos idénticos (Soler, 2002).

Es importante destacar que las presiones de selección surgen, en la mayoría de casos, de manera aleatoria y que los organismos deben sobrevivir a estas con las aptitudes que presenten en el momento en que se presentan, por lo cual no decirles que se iba a evaluar el vuelo del avión resultó bastante útil para la analogía. Los estudiantes que no sabían elaborar aviones de papel fueron eliminados inmediatamente de la carrera, con el fin de que comprendieran que aquellos individuos que no presentan las adaptaciones necesarias para un ambiente y presiones de selección determinadas, son eliminados por la acción de selección natural. Este mensaje fue captado de forma instantánea por el grupo de estudiantes, como se pudo evidenciar en las transcripciones de clase:

Profesor: Antes de que empiecen chicos... ¿Qué pasó con los que no pudieronhacer un avión?

Estudiante: Me morí... ¡Se extinguieron!

Después se les explicó a los estudiantes que los aviones iban a entrar en una competencia y que aquellos que llegaran más lejos serían los ganadores. En este caso, el requerimiento de alcanzar una mayor distancia de vuelo representa la presión de selección. El desempeño en el vuelo de cada avión, reflejado en la distancia que recorre, es una variable análoga que se relaciona con el fitness biológico (aptitud) del individuo, el cual debe entenderse 
como la eficacia que tiene un organismo para sobrevivir y reproducirse, es decir, si el desempeño de vuelo del avión es óptimo, este será seleccionado para "sobrevivir" y "reproducirse".

Tabla 1. Elementos que constituyen el análogo y su relación con los elementos que constituyen el blanco

\begin{tabular}{|c|c|}
\hline Análogo & Blanco \\
\hline Total aviones de papel (20 estudiantes) & Población \\
\hline Avión de papel (por estudiante) & Individuo \\
\hline Diferentes diseños de aviones & Variabilidad intraespecífica \\
\hline Mayor distancia de vuelo & Presión de selección \\
\hline Desempeño en el vuelo & Aptitud (fitness) \\
\hline $\begin{array}{l}\text { Docentes (seleccionan los aviones que alcanzan una mayor } \\
\text { distancia) }\end{array}$ & Selección natural \\
\hline Aviones del primer lanzamiento & Generación F1 (aparece la presión de selección) \\
\hline $\begin{array}{l}\text { Estudiantes que no saben hacer aviones y aquellos que no } \\
\text { alcanzaron la distancia mínima }\end{array}$ & Individuos que no sobreviven \\
\hline Los aviones que mejor vuelan & Individuos más aptos \\
\hline $\begin{array}{l}\text { Los cuatro estudiantes que hicieron los mejores aviones les } \\
\text { enseñan a sus compañeros }\end{array}$ & Reproducción y descendencia de los más aptos \\
\hline $\begin{array}{l}\text { Elaboración de algo novedoso al avión (p. ej. pliegue, } \\
\text { perforación, marca, etc.) }\end{array}$ & $\begin{array}{l}\text { Variabilidad individual y aparición de caracteres al azar (mutaciones) que } \\
\text { pueden o no aumentar la aptitud del individuo (adaptación potencial) }\end{array}$ \\
\hline Caracteres novedosos de los cuatro aviones seleccionados & Adaptación \\
\hline Aviones del segundo lanzamiento & Generación F2 \\
\hline
\end{tabular}

Fuente: elaboración propia.

Siguiendo con el desarrollo de la actividad, los estudiantes lanzaron sus aviones y nosotros seleccionamos los cuatro aviones que mostraron mejor desempeño en el vuelo, es decir, aquellos que alcanzaron una mayor distancia. En este caso, los aviones lanzados son análogos a una generación $\mathrm{F} 1$ y el docente escogió aquellos que llegaron más lejos y descartó los que no superaron la mínima distancia. Esto convirtió al docente en el elemento análogo a la selección natural. Asimismo, estos cuatro aviones seleccionados representan a los individuos más aptos de una generación, aspecto que entendieron los estudiantes rápidamente:

Profesor: ¿Qué creen que pasó ahí?

Estudiante I: Ellos nos ganaron la carrera.

Profesor: ¿Entonces ellos qué son?

Estudiante V: Los más adaptados.
Profesor: Los más adaptados. ¿Y qué va a pasar en la siguiente generación?

Estudiante S: Van a haber muchos más y van a haber menos de nosotros.

Es necesario destacar este aspecto, ya que la analogía permitió a los estudiantes comprender cómo la selección natural elimina a los organismos menos aptos, en consecuencia, los organismos mejor adaptados transmiten su información genética a la siguiente generación. En efecto, la analogía complementó la explicación teórica de la clase, en la cual se mencionó el ejemplo clásico de la evolución del cuello de las jirafas (en un sentido no lamarckiano) y la coloración diferencial de una especie en un entorno bajo presión selectiva de un depredador. En este sentido, la comprensión de la selección natural permite dilucidar la existencia de una variabilidad intraespecífica sujeta a selección diferencial. Entender este efecto combinado, tal como lo explica la teoría sintética, 
además de la concepción de población como un conjunto de individuos representados por un tipo común, sin variaciones, es un problema persistente en el aprendizaje que debe ser abordado, esclarecido y precisado desde la biología evolutiva (Hernández et al., 2009).

Los estudiantes también comprenden que el cambio adaptativo no surge de inmediato en las poblaciones, sino que se da de forma gradual durante varias generaciones, lo que se evidencia en cambios de frecuencias fenotípicas dentro de la población, con la disminución de individuos con el fenotipo limitante en generaciones futuras y el incremento de la proporción de individuos portadores del fenotipo más apto a las condiciones ambientales (Soler, 2002).

Luego, a quienes elaboraron los aviones ganadores se les asignó un grupo de estudiantes con el objetivo de que les enseñaran cómo hacer el avión. La enseñanza por parte de los estudiantes cuyos aviones habían sido los ganadores refleja lo que biológicamente es la reproducción, la cual consiste en la transmisión de información de una generación $\mathrm{F} 1$ a una $\mathrm{F} 2$, en la que los aviones de los estudiantes a quienes se les enseña representan la F2 o la descendencia de los más aptos (F1). A estos mismos estudiantes se les pidió que agregaran algo novedoso al avión de papel (un pliegue, un hueco, un rayón, etc.) antes de iniciar con el lanzamiento, esto con el fin de que los estudiantes comprendieran que en todas las generaciones mientras haya reproducción sexual habrá variabilidad genética, ya sea producto de la recombinación (los estudiantes plasman de alguna manera su forma particular de hacer los aviones aún después de haberse explicado la de los cuatro diseños seleccionados), o producto de la mutación, que es la que corresponde a la característica novedosa que se pidió añadir a cada uno. Ya que no se especificó que la característica que agregaran debía incidir directamente en el desempeño del vuelo, los estudiantes agregaron diferentes atributos, como dibujos, más pliegues, menos pliegues, perforaciones, etc., lo que le dio al análogo el carácter de aleatoriedad presente en la aparición de mutaciones. Después del lanzamiento, ellos evidenciaron que la novedad que habían agregado podía favorecer, perjudicar o no afectar en nada el desempeño en el vuelo de sus aviones, como se evidencia en una de las conclusiones de los estudiantes al final de la actividad:

Profesor: ¿Qué tiene que ver esa novedad? ¿Para qué sirve en la naturaleza?

Estudiante S: Para ser mejor

Estudiante M: Pues si hay una novedad no se sabe si pueda ser algo bueno o no.
Esto es, que la aparición de una característica producto de mutaciones al azar puede aumentar o disminuir el desempeño del vuelo, relacionándose así con un aumento o una disminución del fitness biológico, respectivamente. De igual forma, la mutación puede ser neutra (teoría neutral de la evolución), es decir, que no afecta en nada el desempeño biológico de los individuos, como se dio con aquellas novedades meramente estéticas de los aviones. Así, aquellos caracteres novedosos dentro de una población que se traducen en una mayor probabilidad de supervivencia y reproducción se consideran una adaptación; es decir, aquellas novedades puestas por los estudiantes y que mejoraron de una forma u otra el desempeño en el vuelo de los aviones.

En términos generales, la analogía fue un éxito, ya que permitió a los estudiantes comprender los aspectos básicos de la selección natural, algo que se vio reflejado en la mayoría de las respuestas en la evaluación final, donde usaban la selección natural para explicar un acontecimiento evolutivo particular (aparición de variaciones de aves dentro de una isla, cambios en poblaciones de especies, cambios en las características de las especies a través del tiempo, extinciones, etc.). Para la aplicación de esta o cualquier actividad dirigida a explicar la selección natural es importante tener en cuenta, en primer lugar, que los docentes deben tener un amplio conocimiento del tema, ya que si bien es un tema interesante también es muy complejo por los procesos que integran y subyacen a él. En segundo lugar, se deben tener claros, por lo menos, los niveles organizacionales en los cuales actúan los dos mecanismos principales de cambio evolutivo: la deriva genética y la selección natural, teniendo en cuenta que solo la selección natural causa un cambio adaptativo (Soler, 2002). Sumado a esto, el modelo pedagógico de investigación dirigida requiere de una alta participación del docente durante todo el proceso, por lo que debe establecer una buena comunicación con sus estudiantes y orientarlos activamente en la búsqueda de respuestas y la construcción del conocimiento, brindando las herramientas y el conocimiento necesario sobre la temática (Pozo, 1997).

Se debe destacar que en la ejecución misma de esta analogía nos encontramos con diferentes limitantes; si bien la analogía permite comprender en primera medida la existencia de una variabilidad intraespecífica sobre la que actúa la selección natural en cada población, los conceptos de población y especie sobre los que sienta sus bases la teoría de la evolución (Ruíz Delgado, 2015) no fueron aclarados explícitamente antes ni después del desarrollo, debido a que en la aplicación de la unidad didáctica se contaba con un número de clases limitadas y no se tuvo el tiempo suficiente de abordar dichos conceptos; además, 
dentro de la biología misma aún permanece el debate sobre el significado adecuado de estos conceptos (Mayr, 2006). Esto quiere decir que no hubo una aclaración sobre si los aviones pertenecían o no a la misma especie, qué define a una especie y qué procesos ocurren en este nivel organizacional.

Otra limitante que tiene la analogía es que, aunque explica el proceso de manera general, no incluye aspectos importantes de la selección natural, como la competencia interespecífica, la selección sexual o la depredación. Sin embargo, la analogía funciona como una estrategia complementaria, por lo que estos aspectos pueden abordarse mediante otras herramientas, sin afectar de esta forma al entendimiento del concepto blanco. Asimismo, consideramos que, al no incluir las bases teóricas de la genética mendeliana, la comprensión misma de la segregación de genes es superficial; no obstante, debe tenerse en cuenta que los estudiantes participantes del estudio son de grado séptimo y, considerando los Estándares Básicos de Competencia del Ministerio de Educación Nacional, las bases teóricas de la genética comienzan a abordarse en octavo y noveno. Tampoco se tuvieron en cuenta las diferencias entre adaptación y exaptación, debido a que consideramos que no es necesario hacer esta distinción en este curso, ya que puede hacer un poco más tediosa la comprensión del fenómeno. Entonces, la analogía puede implementarse de forma variable en diferentes cursos de acuerdo con las necesidades y los conocimientos de los estudiantes, de una manera más compleja o incluyendo más elementos en cursos posteriores.

\section{Conclusiones}

La analogía implementada funcionó satisfactoriamente como actividad complementaria de las clases, ya que consiguió que los estudiantes de grado séptimo comprendieran correctamente la evolución por selección natural. Las explicaciones de la selección natural y, en general, de la teoría evolutiva involucran una gran cantidad de conceptos a diferentes niveles organizacionales y que dan sentido a este fenómeno cuando el estudiante logra entenderlos en conjunto. Es importante que la secuencia de las clases y la forma en que se elaboran las unidades didácticas tengan en cuenta la interconexión de los temas y cómo estos se van desarrollando a lo largo del proceso de forma integrada. Así, aunque las analogías sean una herramienta explicativa poderosa dependen de las ideas previamente adquiridas por los estudiantes durante su proceso de aprendizaje. En particular, los estudiantes pueden concebir la selección natural, junto con otros conceptos biológicos, como abstractos o imaginarios; las analogías permiten que los estudiantes visualicen directamente dichos conceptos y los comprendan desde sus bases teóricas.
Dada la dificultad para evidenciar el mecanismo de selección natural de forma experimental en el aula de clase, esta analogía es una herramienta sencilla, rápida, entretenida y de fácil implementación y comprensión, como se evidenció durante el desarrollo de la actividad y en sus explicaciones posteriores. Esta eficacia se debió a que el ejercicio permite establecer una relación clara entre los elementos del análogo y los que constituyen el concepto blanco. Estos conceptos se articulan claramente dentro de la actividad y los alumnos lograron identificarlos y apropiarse de ellos, vinculando así todas las temáticas desarrolladas previamente.

Las analogías son una herramienta muy útil como estrategia complementaria e integradora. Además, pueden funcionar como eje en actividades de transversalización debido a que facilitan la movilización y la comprensión del conocimiento científico, permitiendo así su conexión con otras disciplinas.

\section{Referencias}

Adúriz Bravo, A. (2010). Concepto de modelo científico: una mirada epistemológica de su evolución. En L. Galagovsky (Coord.), Didáctica de las ciencias naturales. El caso de los modelos científicos (pp. 141-161). Lugar Editorial.

Castro Moreno, J. y Valbuena, U. E. (2007). ¿Qué biología enseñar y cómo hacerlo? Hacia una resignificación de la biología escolar. Tecné Episteme y Didaxis: TED, (22), 126-145. https://doi.org/10.17227/ ted.num22-385

Chaves Mejía, G. A. (2012). Contribuciones a la enseñanza de la evolución biológica desde la revisión epistemológica de algunos aspectos contemporáneos de la misma [Tesis de maestría, Universidad Nacional de Colombia]. Repositorio institucional UnAL https://repositorio.unal.edu.co/handle/unal/11169

Chaves Mejía, G. A. (2016). La enseñanza y el aprendizaje de la evolución biológica con la perspectiva teórica del perfil conceptual: implicaciones en la formación continua del profesorado. Bio-grafía, 9(17), 109-117.

Gagliardi, R. (1986). Los conceptos estructurantes en el aprendizaje por investigación. Enseñanza de las Ciencias, 4(1), 30-35.

Glynn, S. M. y Takahashi, T. (1998). Learning from analogy-enhanced science text. J. Res. Sci. Teach., 35, 1129-1149. https://doi.org/10.1002/(sICI)10982736(199812)35:10<1129::AID-TEA5>3.0.CO;2-2 
González Galli, L. y Meinardi, E. (2015). Obstáculos para el aprendizaje del modelo de evolución por selección natural, en estudiantes de escuela secundaria de Argentina. Ciência y Educação, 21(1), 101-122. https://dx.doi.org/10.1590/1516-731320150010007

Hernández R., M. C., Álvarez Pérez, E. y Ruiz Gutiérrez, R. (2009). La selección natural: aprendizaje de un paradigma. Teorema, 28(2), 107-121.

Jiménez A., M. P. (1991). Cambiando las ideas sobre el cambio biológico. Enseñanza de las ciencias, 9(3), 248-256.

Marcos Merino, J. M., Gallego, R. E. y Ochoa, J. E. (2017). Comprendiendo los relojes moleculares a través de la evolución de El Quijote. Enseñanza de las Ciencias (número extraordinario), 3753-3758.

Martínez Arango, A. V. y Sanabria Totaitive, I. A. (2018). Evolución de los videojuegos: Analogía como estrategia para enseñar evolución biológica. Tecné, Episteme y Didaxis: TED, Número extraordinario.

Mayr, E. (2006). ¿Por qué es única la Biología? Consideraciones sobre la autonomía de una disciplina científica. Katz.

Naranjo Cardona, L. C. (2013). Diseño de una unidad didáctica para la enseñanza de la evolución. [Tesis de maestría, Universidad Nacional de Colombia]. Repositorio institucional unaL https://repositorio. unal.edu.co/handle/unal/20383

Oliva, J. M. (2007). Qué conocimientos profesionales deberíamos tener los profesores de ciencias sobre el uso de analogías. Revista Eureka sobre Enseñanza y Divulgación de las Ciencias, 5(1), 15-28.

Oliva, J. M.; Aragón, M. M.; Mateo, J. y Bonat, M. (2001). Una propuesta didáctica basada en la investigación para el uso de analogías en la enseñanza de las ciencias. Enseñanza de las Ciencias, 19(3), 453-470.

Oliva, J. M., Aragón, M. M., Bonat, M. y Mateo, J. (2003). Un estudio sobre el papel de las analogías en la construcción del modelo cinético-molecular de la materia. Enseñanza de las Ciencias, 21(3), 429-444.

Oliva, J. M. y Aragón, M. M. (2009). Contribución del aprendizaje con analogías al pensamiento modelizador de los alumnos en ciencias: marco teórico. Enseñanza de las Ciencias, 27(2), 195- 208.

Pozo, J. I. (1997). Enfoques para la enseñanza de la ciencia. En Teorías cognitivas del aprendizaje (pp. 265-308). Morata. Ruíz Delgado, A. (2015). Banco de actividades para trabajar ideas previas y dificultades en el aprendizaje y la enseñanza de la evolución [Tesis de maestría, Universidad de Granada]. Repositorio institucional UGR. http://hdl. handle.net/10481/38448

Soler, M. (2002). Selección natural y adaptación. En M. Soler (Ed.), Evolución, la base de la biología. (pp. 127-118). Proyectur Sur de Ediciones.

Van Dijk, E. M. y Reydon, T. A. C. (2010). A conceptual analysis of evolutionary theory for teacher education. Science and Education, 19(6-8), 655-677. 